\title{
PRELIMINARY RESULTS OF RELATIVE DECLINATION OBSERVATIONS MADE WITH THE REPSOLD MERIDIAN CIRCLE AT CERRO CALÁN
}

\author{
G. CAR RASCO
}

Departamento de Astronomia, Universidad de Chile, Chile

\begin{abstract}
Preliminary results obtained from 6 years of declination observations are presented. Some instrumental details are discussed, and the observed declination system is compared with FK4 in $\Delta \delta_{\delta}$.
\end{abstract}

During the observations of the Southern Reference Stars and Bright Stars Programme with the Repsold Meridian Circle $(d=19 \mathrm{~cm}, F=224 \mathrm{~cm})$ at Cerro Calán Observatory, series of observations were made, along the whole meridian arc, of stars of the Fundamental Catalogue FK4. This paper gives the preliminary results in declination of the observations made from January 1963 to December 1968.

Four observers carried out 52 series, with a total number of 1721 individual observations of stars of the FK4 Catalogue with declination from $+41^{\circ}$ to $-90^{\circ}$ in upper culmination and from the pole to $-68^{\circ}$ in lower culmination (Table I). This material represents about $40 \%$ of the total number of observations made by ten observers during this period.

About $20 \%$ of the series were made with visual circle readings and for the other $80 \%$, FOLM photographic cameras (four on each side) were used to take photographs of the circle readings.

All the films were measured by the author in the Pulkovo Observatory, with the semi-automatic photo-electric measuring machine designed by Dr Platonov.

Before the start of the observations, the screw of the eye-piece micrometer and the screws of the four microscope-micrometers for the visual circle reading were investigated by the chain method. For the determination of periodic errors, the Rydberg method was used with an interval of $\frac{3}{5}$ and $\frac{3}{4}$ of revolution. For the eye-piece micro-

TABLE I

Distribution of the observations by observers

\begin{tabular}{|c|c|c|c|c|c|c|}
\hline \multirow[t]{2}{*}{ Observer } & \multirow{2}{*}{$\begin{array}{l}\text { Number } \\
\text { of series }\end{array}$} & \multicolumn{3}{|c|}{ Number of observations } & \multicolumn{2}{|c|}{ Mean errors } \\
\hline & & UC & LC & Total & $E_{0}$ & Observation \\
\hline Anguita & 8 & 198 & 29 & 227 & $\pm 0 " 476$ & \pm 0.497 \\
\hline Carrasco & 31 & 895 & 151 & 1046 & 0.449 & 0.541 \\
\hline Loyola & 8 & 283 & 29 & 312 & 0.452 & 0.542 \\
\hline Mercado & 5 & 123 & 13 & 136 & 0.516 & 0.560 \\
\hline Totals & 52 & 1499 & 222 & 1721 & \pm 0.473 & \pm 0.535 \\
\hline
\end{tabular}


meter screw the periodic error has a maximum value of 0.07 (equal to $0.004 R$ ). The progressive error is practically the same for all the screws. The screws of the microscope-micrometers show a very small periodic error.

The value of one revolution of the declination screw was determined several times during these years with the mercury mirror. The value adopted was $R=18^{\prime \prime} 38$.

For the investigation of the diameter errors of the main circle the Bruns method was used. The corrections of the circle reading were determined for an interval of $3^{\circ}$ using three rosettes $R(3, x), R(4, x)$ and $R(5, x)$. The systematic error is about 0.4 and the individual corrections go from +0.45 to -0.35 for the four microscopes. The centring error of the main circle corresponds to about $19^{\prime \prime}$ of the circle and its inclination from the plane normal to the rotation axis is 0.9 (Anguita et al., 1963).

A second investigation of the corrections of all diameters (each 4'), using the BrunsLevy photographic method (Zverev, 1964) was carried out at the end of 1966 according to the following schema:

$$
R(2700, x)=R(45, x) \times R(6, x) \times R(10, x) .
$$

For this purpose, four photographic cameras installed in the East cage were used, in which the normal indexes were replaced by new ones with 12 divisions at a spacing equal to that of the circle graduations.

The main object of the investigation, the measure of the rosette $R(45, x)$, was done with six angles between the diameters $\left(36^{\circ}, 72^{\circ}, 20^{\circ}, 60^{\circ}\right.$ and $\left.80^{\circ}\right)$ and for the reduction of all the measures to the main system, two sets of exposures were taken; for the first coordination, angles $28^{\circ} 40^{\prime}, 29^{\circ} 20^{\prime}$ and $30^{\circ} 00^{\prime}$ were used, and for the second coordination, $67^{\circ} 28^{\prime}, 67^{\circ} 36^{\prime}$ and $67^{\circ} 40^{\prime}$.

Nearby 4000 exposures (16000 pictures) were taken and the measures of this film, with the new automatic measuring machine designed in Odessa, are in progress in the Pulkovo Observatory.

The reductions of the observations were made with the IBM-360 computer of the University of Chile, using the following formulae:

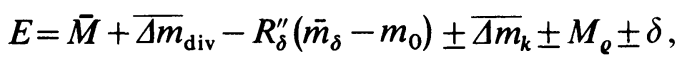

where the upper sign is for clamp East and the lower for clamp West ; $E$, is the equator point; $\bar{M}$, the mean reading for the four microscopes; $\overline{\Delta m}_{\mathrm{div}}$, the correction for the circle reading (preliminary results obtained in 1962); $R_{\delta}^{\prime \prime}$, the values of one revolution for the declination screw; $\bar{m}_{\delta}$, mean of the four readings of the declination screw; $m_{0}=4000 ; \overline{\Delta m}_{k}$, correction for the curvature of parallel; $M_{e}$, correction for refraction (computed from the Pulkovo Refraction Tables) and $\delta$, the apparent declination of the FK4 stars. This formula was used for upper culmination; for lower culmination $\delta$ was replaced by $|\delta|-180^{\circ}$.

For each series the equator point $E_{0}$ was determined with stars whose zenith distance is $|Z|<30^{\circ}$. More than $80 \%$ of the observations show a large rate of the equator point with time. With the zenith stars a linear rate of the equator point was 
determined and with these parameters the equator point $E_{0}$ for each observed star is computed and the difference $\Delta E=E-E_{0}$ is obtained.

Only three series show anomalies in the rate of the equator point with time and they were reduced by a graphical method.

On the average, the mean error of one determination of the equator point $E_{0}$ is \pm 0 ". 462 and the mean error of one observation is \pm 0 ".540.

The differences $\Delta E$ were grouped in zones of $5^{\circ}$ and the mean values $\overline{\Delta E}$ for each zone were computed. All the observations with $\Delta E$ differing by more than 1 ".00 from the mean value were excluded after a careful examination of the observing data. The mean error for one difference $\Delta E$ is \pm 0 ".463 for upper culmination and \pm 0 ".500 for lower culmination.

These values, slightly smoothed, are given in Table II for the two positions of the instrument, and are plotted in Figure 1. This figure represents 908 values for clamp E and 637 for clamp W.

TABLE II

Mean values $\overline{\Delta E}$ in both positions of the instrument, for $5^{\circ}$ declination zone

(Unit:0:01)

\begin{tabular}{|c|c|c|c|c|c|}
\hline \multirow{2}{*}{$\delta$} & \multicolumn{2}{|l|}{$\overline{\Delta E}$} & \multirow{2}{*}{$\delta$} & \multicolumn{2}{|l|}{$\overrightarrow{\Delta E}$} \\
\hline & $\mathrm{E}$ & W & & $\mathbf{E}$ & W \\
\hline$+40^{\circ}$ & +18 & -42 & $-40^{\circ}$ & -18 & -10 \\
\hline+35 & -07 & -39 & -45 & -04 & -11 \\
\hline+30 & +07 & -35 & -50 & -06 & +03 \\
\hline+25 & +40 & -13 & -55 & -17 & -08 \\
\hline+20 & +34 & +17 & -60 & +04 & +07 \\
\hline+15 & +38 & +15 & -65 & -07 & +43 \\
\hline+10 & +43 & -10 & -70 & -40 & +63 \\
\hline+05 & +30 & -14 & -75 & -31 & +68 \\
\hline 00 & +16 & -08 & -80 & +01 & +50 \\
\hline-05 & +08 & -02 & -85 & -05 & +18 \\
\hline-10 & +16 & +03 & -90 & -32 & +30 \\
\hline-15 & +07 & +10 & $-85 \mathrm{sp}$ & -48 & +42 \\
\hline-20 & -04 & +04 & $-80 \mathrm{sp}$ & -31 & +35 \\
\hline-25 & +01 & -03 & $-75 \mathrm{sp}$ & -38 & +10 \\
\hline-30 & +06 & -05 & $-70 \mathrm{sp}$ & -12 & -02 \\
\hline-35 & -04 & +02 & & & \\
\hline
\end{tabular}

In this figure it is possible to see the rate in declination of the $\Delta E$ and also the horizontal flexure, given by $f=b \sin z$; there is a large deviation in the clamp W position between $-65^{\circ}$ to $-80^{\circ}$, probably due to errors in the first determination of the circle diameters corrections; this deviation appears for clamp $\mathrm{E}$ in the symmetrical position in zone $+5^{\circ}$ to $+20^{\circ}$.

In order to determine if this deviation is real for the Repsold Meridian Circle, the $\Delta E$ values were computed for the main observers: the results are given in Table III. The points in the zone $-65^{\circ}$ to $-80^{\circ}$ for clamp $\mathrm{W}$ show very good agreement. 


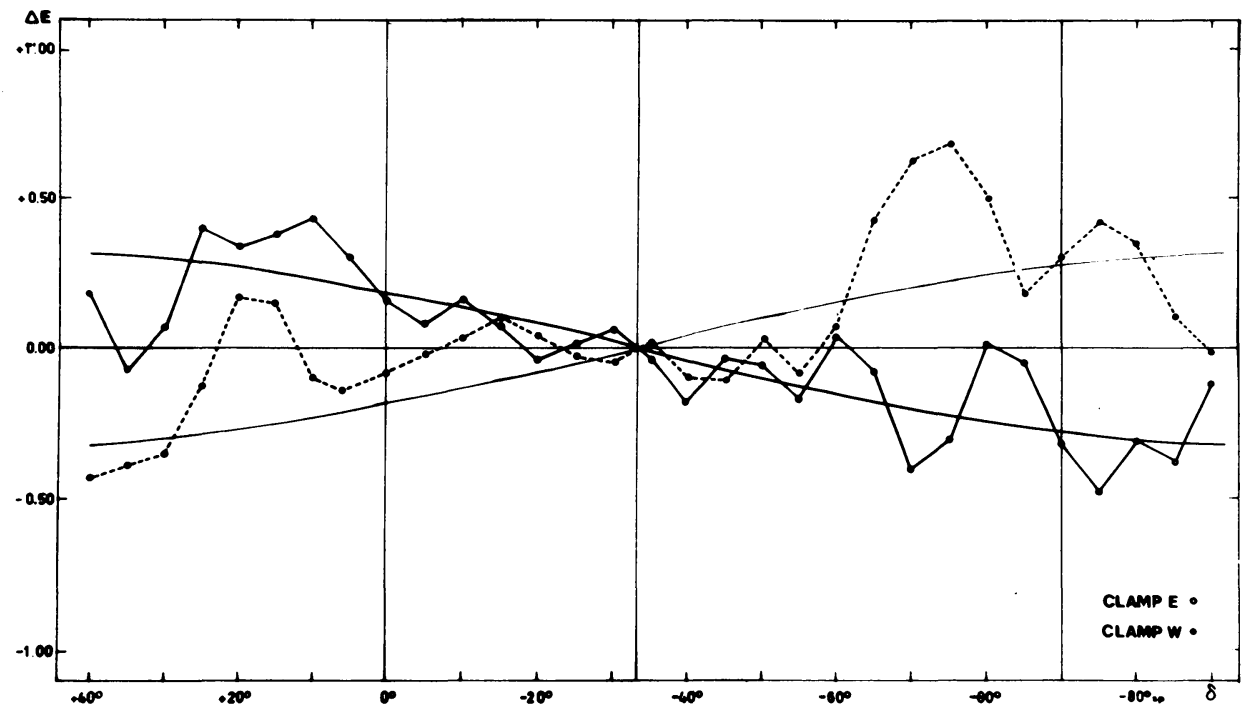

Fig. 1. Mean values $\overline{\Delta E}$ for the two positions of the instrument. (Slightly smoothed.)

TABLE III

Mean values $\overline{\Delta E}$ in both positions of the instrument by observers (Unit:0"01)

\begin{tabular}{|c|c|c|c|c|c|}
\hline \multirow{2}{*}{$\delta$} & \multicolumn{3}{|l|}{ Clamp E } & \multicolumn{2}{|l|}{ Clamp W } \\
\hline & Anguita & Carrasco & Loyola & Carrasco & Loyola \\
\hline$+40^{\circ}$ & -08 & +32 & - & -51 & - \\
\hline+35 & -29 & +01 & -39 & -50 & -32 \\
\hline+30 & +29 & +19 & -37 & -52 & -43 \\
\hline+25 & +32 & +54 & -05 & -37 & 00 \\
\hline+20 & +22 & +38 & +20 & -11 & +37 \\
\hline+15 & +39 & +34 & +43 & -02 & +29 \\
\hline+10 & +45 & +41 & +24 & -13 & 00 \\
\hline+05 & +30 & +28 & +18 & -26 & +15 \\
\hline 00 & +07 & +11 & +35 & -24 & +27 \\
\hline-05 & +14 & +02 & +25 & -17 & +07 \\
\hline-10 & +45 & +07 & +16 & -02 & +01 \\
\hline-15 & +33 & +06 & -10 & +14 & +29 \\
\hline-20 & -04 & +01 & -18 & +04 & +20 \\
\hline-25 & 00 & +03 & +06 & -01 & -09 \\
\hline-30 & +17 & +08 & -06 & -01 & -09 \\
\hline-35 & +03 & -02 & -28 & +04 & -07 \\
\hline-40 & -19 & -17 & -17 & -07 & -10 \\
\hline-45 & -11 & -04 & +03 & -13 & +02 \\
\hline-50 & -12 & -11 & +25 & +02 & +16 \\
\hline-55 & -46 & -14 & +05 & -06 & -03 \\
\hline-60 & -35 & +12 & +22 & +10 & +06 \\
\hline-65 & -10 & -14 & +22 & +48 & +35 \\
\hline-70 & -21 & -49 & -28 & +67 & +64 \\
\hline
\end{tabular}


Table III (Continued)

\begin{tabular}{lrrrrr}
-75 & -36 & -28 & -35 & +69 & +70 \\
-80 & -13 & +04 & -02 & +50 & +48 \\
-85 & -09 & -02 & -14 & +02 & +25 \\
-90 & -33 & -38 & +15 & +29 & -03 \\
$-85 \mathrm{sp}$ & -30 & -53 & - & +53 & +33 \\
$-80 \mathrm{sp}$ & -48 & -30 & - & +45 & +42 \\
$-75 \mathrm{sp}$ & -56 & -40 & - & +13 & +11 \\
$-70 \mathrm{sp}$ & - & -14 & - & -17 & +25 \\
\hline
\end{tabular}

TABLE IV

Systematic differences of the type $\Delta \delta_{\delta}$ in the sense Repsold Meridian Circle - FK4 (Unit:0"01)

\begin{tabular}{rlll}
\hline$\delta$ & $\Delta \delta_{\delta}$ & $\delta$ & $\Delta \delta_{\delta}$ \\
\hline$+40^{\circ}$ & -12 & $-40^{\circ}$ & -14 \\
$+35^{\circ}$ & -23 & $-45^{\circ}$ & -08 \\
$+30^{\circ}$ & -14 & $-50^{\circ}$ & -02 \\
$+25^{\circ}$ & +14 & $-55^{\circ}$ & -13 \\
$+20^{\circ}$ & +25 & $-60^{\circ}$ & +05 \\
$+15^{\circ}$ & +26 & $-65^{\circ}$ & +18 \\
$+10^{\circ}$ & +16 & $-70^{\circ}$ & +11 \\
$+05^{\circ}$ & +08 & $-75^{\circ}$ & +19 \\
00 & +04 & $-80^{\circ}$ & +26 \\
-05 & +03 & -85 & -06 \\
-10 & +10 & -90 & -01 \\
-15 & +08 & $-85 \mathrm{sp}$ & -03 \\
-20 & -02 & $-80 \mathrm{sp}$ & +02 \\
-25 & -01 & $-75 \mathrm{sp}$ & -14 \\
-30 & +01 & $-70 \mathrm{sp}$ & -07 \\
-35 & -01 & & \\
\hline
\end{tabular}

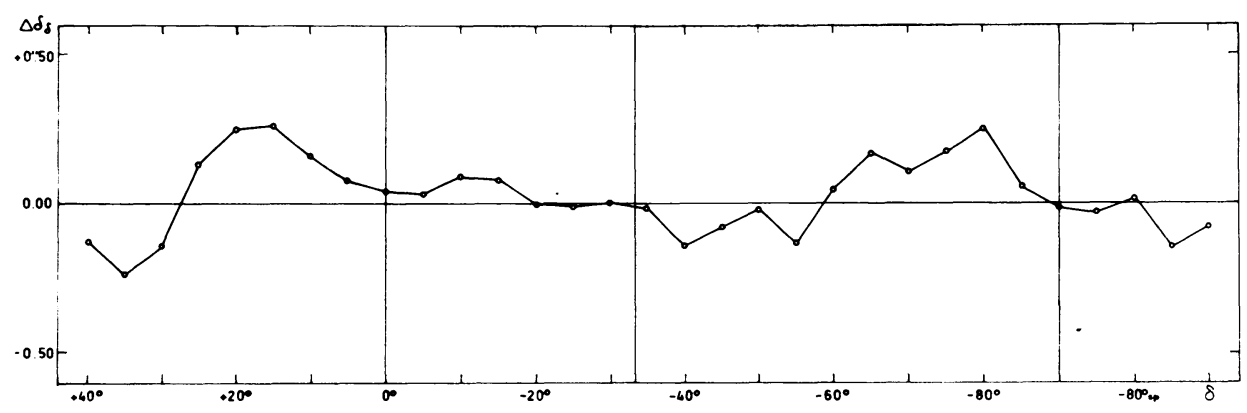

Fig. 2. Systematic differences $\Delta \delta_{\delta}$ in the sense Repsold Meridian Circle -FK4. 
Finally the $\frac{1}{2}(E+W)$ values were computed, representing the systematic differences $\Delta \delta_{\delta}$ in the sense Repsold Meridian Circle - FK4. The results are given in Table IV and Figure 2. The deviation of the curve in the southern declinations is probably real. For northern declinations this deviation is probably due to refraction anomalies.

\section{Acknowledgement}

The author wishes to thank the staff of the Astronomy Department, Observatorio Astronomico, Cerro Calán, for their help in this work.

\section{References}

Anguita, C., Bagildinsky, B. K., Carrasco, G., Loyola, P., Shishkina, V. N., and Zverev, M. S.: 1963 , Inf. Bull. South. Hemisph. 3, 30.

Zverev, M. S. : 1964, Astron. Zh. 41, 1128 (1965, Soviet Astron. 8, 897).

\section{DISCUSSION}

Wood: The results of Dr Carrasco reveal an important point about the surroundings in which a transit instrument should be sited.

Dieckvoss: Did you measure the circle photographically all the time?

Carrasco: The circle was measured visually for $20 \%$ of the observations, and photographically for the other $80 \%$.

The general results in both cases are equal. 